(Aus dem physiologischen Institut der Universität Lemberg.)

\title{
Versuche über die sensorische Funktion des Kleinhirnmittelstücks (Vermis) ${ }^{1}$ ).
}

\author{
Von \\ Prof. Dr. A. Beck und Prof. Dr. G. Bikeles.
}

(Mit 2 Textfiguren.)

In vorliegender Arbeit stellten wir uns zur Aufgabe die Lösung folgender Fragen:

1. Lassen sich bei Reizung irgend eines peripheren Nerven Aktionsströme vom Mittelstück des Kleinhirns (Vermis), zu dem bekanntlich die spinocerebellaren Bahnen hinziehen, erhalten?

2. Wie ist das Verhältnis etwaiger solcher Aktionsströme zu denen von der psychomotorischen Region bei Reizung desselben peripheren Nerven erhaltenen?

3. Lässt sich bei Reizung verschiedener peripherer Nerven für das Erhalten von Aktionsströmen an verschiedenen Bezirken des Kleinhirnmittelstückes irgend eine sensible Lokalisation konstatieren?

Das Versuchsverfahren war hierbei folgendes:

Beim kuraresierten Hunde wurde die hintere Kleinhirnfläche und nebst dem eine kleine Partie am Hinterhauptlappen der einen Seite, in einer Reihe von Fällen auch die psychomotorische Region der anderen Seite blossgelegt und unter einen entsprechenden peripheren Nerv (meistens Ischiadicus), am häufigsten auf der dem blossgelegten Hinterhauptlappenteile gleichen Seite, Reizelektroden untergeschoben. Hierauf wurde die Rinde des Kleinhirnmittelstückes (Lobus med. post. Bolk, Vermis posterior) und als indifferente Stelle die Hirnrinde des Hinterbauptlappens vermittels unpolarisierbarer Elektroden mit dem Galvanometer verbunden.

1) Vorgelegt der Lrakauer Akademie der Wissenschaften in der Sitzung vom 6. Oktober 1911, erscheint in polnischer Sprache in den Abhandlungen der Akademie Bd. $51 \mathrm{~B}$. 
Auf den Vermis wurde überwiegend der als Elektrodenende dienende Wollfaden quer in frontaler Richtung aufgelegt 1). (S. Fig. 1.)

Hierauf wurde der entsprechende Nerv mehrfach hintereinander mit schwachen Induktionsströmen gereizt und die jedesmalige Galvanometerablenkung beobachtet und notiert in derselben Weise, wie es in der vorangegangenen Arbeit ausführlich angegeben wurde.

In den ersten Versuchen gelang uns nicht eine Negativität der Kleinhirnrinde bei solchen Reizungen nachzuweisen, wabrscheinlich weil die Kleinhirnrinde trotz des makroskopisch normalen Aussehens vielleicht doch während des Blosslegens irgendwie gelitten hat. Hin= gegen konnten wir bei einer enormen Zahl der später nachfolgenden Versuche ein reichliches Auftreten von Aktionsströmen am Kleinhirnmittelstück bei Reizung des peripheren Nerven konstatieren.

In sehr vielen Versuchen wurde zum Vergleich bei Reizung eines und desselben Nerven abwechselnd vom Hinterwurm und von der dem gereizten Nerven kontralateralen psychomotorischen Region abgeleitet. Das Ergebnis, welches in der Tabelle I übersichtlich dargestellt ist, war, dass im grossen und ganzen die Aktionsströme an der psychomotorischen Region etwas häufiger

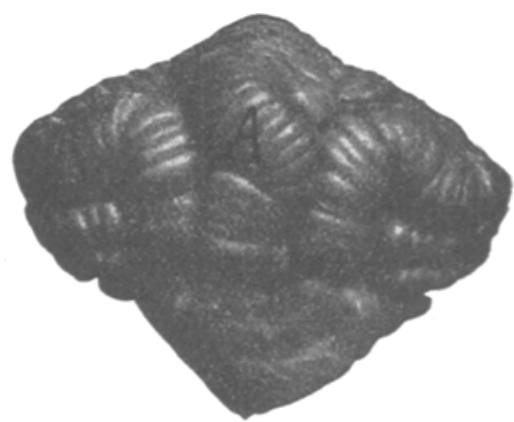

Fig. 1. und intensiver zum Vorschein kamen als am Vermis. Jedoch war in manchen Fällen ein Untersehied kaum vorhanden (Versuchszahl 5,6), und in zwei Fällen zeigten sich die Aktionsströme gerade am Kleinhirn etwas beträchtlicher (Versuchszahl 8 und 10).

In einer Reihe von Versuchen wurden abwechselnd mit dem Ischiadicus auch Nerven vom Plexus brachialis (meist Medianus und Ulnaris) derselben Seite mit schwachen Induktionsströmen gereizt und zunächst ebenfalls vom Vermis poster. abgeleitet. Nebst dem waren wir auch bestrebt, sowohl bei Reizung des Ischiadicus als

1) In einer Minderheit von Versuchen wurde auch der Elektrodenfaden einseitig in sagittaler Richtung, manchmal auch punktförmig auf den Vermis aufgelegt; doch zogen wir das Auflegen in frontaler Richtung (beiderseits von der Medianlinie) - da uns die Kreuzungsverhältnisse der afferenten Bahnen weniger interessierten - vor. 
Tabelle I.

Zum Vergleich der Aktionsströme erhalten: a) vom Vermis posterior, b) von der psychomotorischen Region. Reizung eines peripheren Nerven.

\begin{tabular}{|c|c|c|c|c|c|c|c|c|c|c|}
\hline \multirow{3}{*}{ 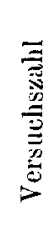 } & \multicolumn{5}{|c|}{ Ableitung vom Vermis } & \multicolumn{5}{|c|}{$\begin{array}{c}\text { Ableitung von der psychomotorischen } \\
\text { Region }\end{array}$} \\
\hline & \multirow{2}{*}{$\begin{array}{c}\mathrm{Ge}- \\
\text { reizt } \\
\text { wurde } \\
\text { mal }\end{array}$} & \multicolumn{3}{|c|}{$\begin{array}{l}\text { Negativwerden des } \\
\text { Kleinhirns }\end{array}$} & \multirow{2}{*}{ 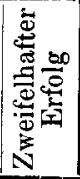 } & \multirow{2}{*}{$\begin{array}{c}\text { Ge- } \\
\text { reizt } \\
\text { wurde } \\
\text { mal }\end{array}$} & \multicolumn{3}{|c|}{$\begin{array}{l}\text { Negativwerden der } \\
\text { psychomotor. Region }\end{array}$} & \multirow{2}{*}{ 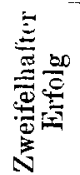 } \\
\hline & & mal & $\begin{array}{l}\text { in } \\
\text { Proz. }\end{array}$ & $\begin{array}{l}\text { Grösse der } \\
\text { Ablenkung } \\
\text { in škalent. }\end{array}$ & & & mal & $\begin{array}{l}\text { in } \\
\text { Proz. }\end{array}$ & $\begin{array}{c}\text { Grösse der } \\
\text { Ablenkung } \\
\text { in Skalent. }\end{array}$ & \\
\hline I & 8 & 5 & 62.5 & $7-10$ & 2 & 4 & 4 & 100 & $13-25$ & \\
\hline 2 & 11 & 5 & 45,4 & $10-13$ & 2 & 8 & 5 & 62,5 & $10-17$ & 2 \\
\hline 3 & 10 & 6 & $60^{2}$ & $5-8$ & & 10 & 7 & $70^{\circ}$ & $17-23$ & \\
\hline 4 & 9 & 5 & 55,5 & $7-1 \frac{1}{1}$ & 1 & 8 & $\dot{5}$ & 62,5 & $5-15$ & \\
\hline 5 & 8 & 5 & 62, & $7-9$ & & 7 & 5 & 62 & $12-19,5$ & \\
\hline 6 & 7 & 5 & 71 & $8-10,5$ & 1 & 4 & 3 & 75 & $12-18$ & 1 \\
\hline 7 & 6 & 4 & 66,6 & $6,5-9,5$ & 1 & 6 & 5 & 8 & $5-16$ & 1 \\
\hline & 5 & 4 & $80^{\circ}$ & $5-9$ & 1 & 6 & 4 & 67 & $5-12$ & 1 \\
\hline & 8 & 6 & 75 & $6-8$ & 1 & 5 & 5 & 100 & $3-11$ & \\
\hline 1 & 8 & 4 & 50 & $4--11$ & 1 & 7 & 2 & 30 & $5-7$ & \\
\hline 11 & 10 & 7 & 70 & $7-16,5$ & & 7 & 6 & 89 & $9-39$ & 1 \\
\hline
\end{tabular}

auch bei der der Armnerven (Medianus und Ulnaris), das etwaige Auftreten von Aktionsströmen an der oberen Fläche des Vermis zu studieren und deren Verhalten bei abwechselnder Reizung erwähnter Nerven festzustelleı.

Da das Anlegen des Wollfadens der inpolarisierbaren Elektrode an der oberen Vermisfläche - ohne Berührung des Nachbargewebes unmöglich war, bedienten wir uns zu diesem Zwecke unpolarisierbarer Chlorsilberelektroden. Dieselben bestanden aus $40 \mathrm{~mm}$ langen, $3 \mathrm{~mm}$ breiten glattpolierten, etwa $1 \mathrm{~mm}$ dicken Silberstreifen von entsprechender Krümmung, welche durch ihre ganze Läıge mit einer ziemlich dicken Chlorsilberschichte bedeckt waren. Die ganze Oberfläche der Elektrode war vermittels eines Lackes isoliert bis auf eine $3 \mathrm{~mm}$ lange Stelle am Ende der Elektrode und zwar an der Fläche, welche mit der zu untersuchenden Stelle des Vermis in Berührung kommen sollte. Während die eine von dem Elektrodenpaare auf den Hinterhauptlappen angelegt wurde, wurde die andere, mit der Concavität zum Kleinhirn gewendet, behutsam zwischen dasselbe und das Tentorium je nach Bedarf verschieden tief hineingeschoben. Bei jeder derartigen Elektrodeneinschiebung wurde das herausragende Stück der Elektrode genau gemessen und notiert; nach Ablauf des Versuches wurde wiederum durch abermalige An- 
Versuche über die sensor. Funktion des Kleinhirnmittelstücks (Vermis). 299

legung der Elektrode an den gänzlich freigelegten Vermis die während des Versuches mit dem nichtisolierten Elektrodenende in Berührung gekommene und abgeleitete Stelle markiert. Auf diese Weise wurde in verschiedenen Versuchen fast die ganze Länge der oberen Fläehe des Vermis abgetastet.

Die Ergebnisse dieser Versuche veranschaulichen die in Tabelle II in Kürze beigefügten Prozentzahlen, betreffend das Auftreten von Aktionsströmen während der Reizung der peripheren Extremitätennerven, d. i.: des N. Ischiadicus einerseits und der Armnerven andererseits.

Tabelle II.

Häufigkeit der Aktionsströme in Prozenten.

\begin{tabular}{c|r|c|c|c|c|c}
\hline \hline & \multicolumn{3}{|c|}{$\begin{array}{c}\text { Reizung } \\
\text { des Ischiadicus }\end{array}$} & \multicolumn{3}{|c|}{$\begin{array}{c}\text { Reizung } \\
\text { der Armnerven }\end{array}$} \\
\cline { 2 - 7 } & Max. & Min. & Mittel & Max. & Min. & Mittel \\
\hline $\begin{array}{c}\text { Ableitung vom Hinterwurm . } \\
\text { Ableitung von der oberen }\end{array}$ & 100 & 50 & 69 & 100 & 50 & 67 \\
Fläche des Wurmes . - . & 85 & 43 & 62 & 100 & 30 & 56
\end{tabular}

Man ersieht daraus, dass Reizung von Nerven sowohl der hinteren als auch der vorderen Extremität das Erhalten von Aktionsströmen in fast gleicher Häufigkeit und Intensität gestattet. Ein Überwiegen der Aktionsströme, abbängig davon, ob ein Nerv einer hinteren oder einer vorderen Extremität gereizt wurde, ist weder bei Ableitung von der hinteren, noch von der oberen Fläche des Vermis vorhanden. Bemerken wollen wir an dieser Stelle, dass von der hinteren Vermispartie die Ableitung von der Gegend der

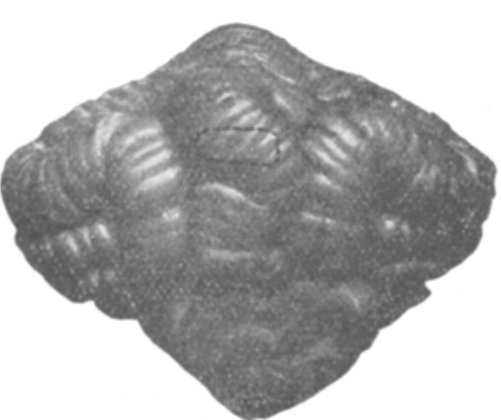

Fig. 2. beim Hunde nach rechts gerichteten Curvatur (Fig. $2 A$ ) im allgemeinen sowohl bei Reizung der Nerven der vorderen als auch der hinteren Extremitäten gewöhnlich bessere Aktionsströme erhalten liess als von der unterhalb derselben befindlichen Partie des Vermis posterior. 
Besonders hervorheben wollen wir noch, dass Reizung der Nn. vagi ebenfalls vom ganzen uns zugänglichen Kleinhirnmittelstück unzweifelhafte Aktionsströme, wenn auch im allgemeinen in einem etwas schwächeren Grade als bei Reizung der Extremitätennerven erhalten lässt und zwar in Prozenten ausgedrückt bei Ableitung vom Vermis posterior: Maximum 85, Minimum 37,5, Mittel 46,6, bei Ableitung wiederum von der oberen Fläche des Mittelstücks: Maximum 87,5, Minimum 30, Mittel 53. Dies verdient um so mehr Beachtung, als wir bei wiederholten Versuchen einer Ableitung von der Grosshirnrinde (d. i. von der psychomotorisehen und der angrenzenden Region) bei Reizung des N. vagus Aktionsströme gänzlich vermissten. Nebstbei liefert dies eine physiologische Bestätigung der Angabe Edinger's, wonach vom Kern des N. vagus sekundäre Bahnen an den Vermis cerebelli gelangen.

Das Auftreten nun von Aktionsströmen an jeder beliebigen, überhaupt irgendwie zugänglichen Partie des Kleinhirnmittelstückes bei Reizung von Nerven der hinteren, der vorderen Extremität und sogardes N. vagus spricht entschieden gegen jede sensible Lokalisation am Vermis.

Auch Mac Nalty und Horsley ${ }^{1}$ ) gelangen auf Grund anatotomischer Untersuchungen zum Ergebnis, dass es eine sensible Lokalisation im Kleinhirn (korrekt sollte es eigentlich lauten im Kleinbirnmittelstück) nicht gibt. Doch behalten wir uns die Würdigung anatomischer Verhätnisse und deren Vergleich mit den Ergebnissen physiologischer Untersuchungen für spätere Arbeiten vor.

Im Verlaufe der obigen Untersuchungen wurde in acht Versuchen (vgl. Tab. III, S. 301 und 302) bei Reizung eines Extremitätennerven nach Ableitung vom Lobus medianus posterior (Vermis posterior) eine solche noch vom Lobulus paramedianus Bolk, also schon im Bereich der Hemisphäre, vorgenommen.

In zwei Versuchen erhielten wir wohl vom Vermis Aktionsströme, nicht aber von der Hemisphäre; in vier Versuchen waren die am Paramedianus beobachteten Aktionsströme bedeutend seltener

1) Mac Nalty and Horsley, Brain vol. 32 p. 237 . On the cervical spino-bulbar and spino-cerebellar tracts and on the question of topographical representation in the cerebellum. 
Versuche über die sensor. Funktion des Kleinhirnmittelstücks (Vermis). 301

Tabelle III.

Reizung peripherer Nerven rechtseitig.

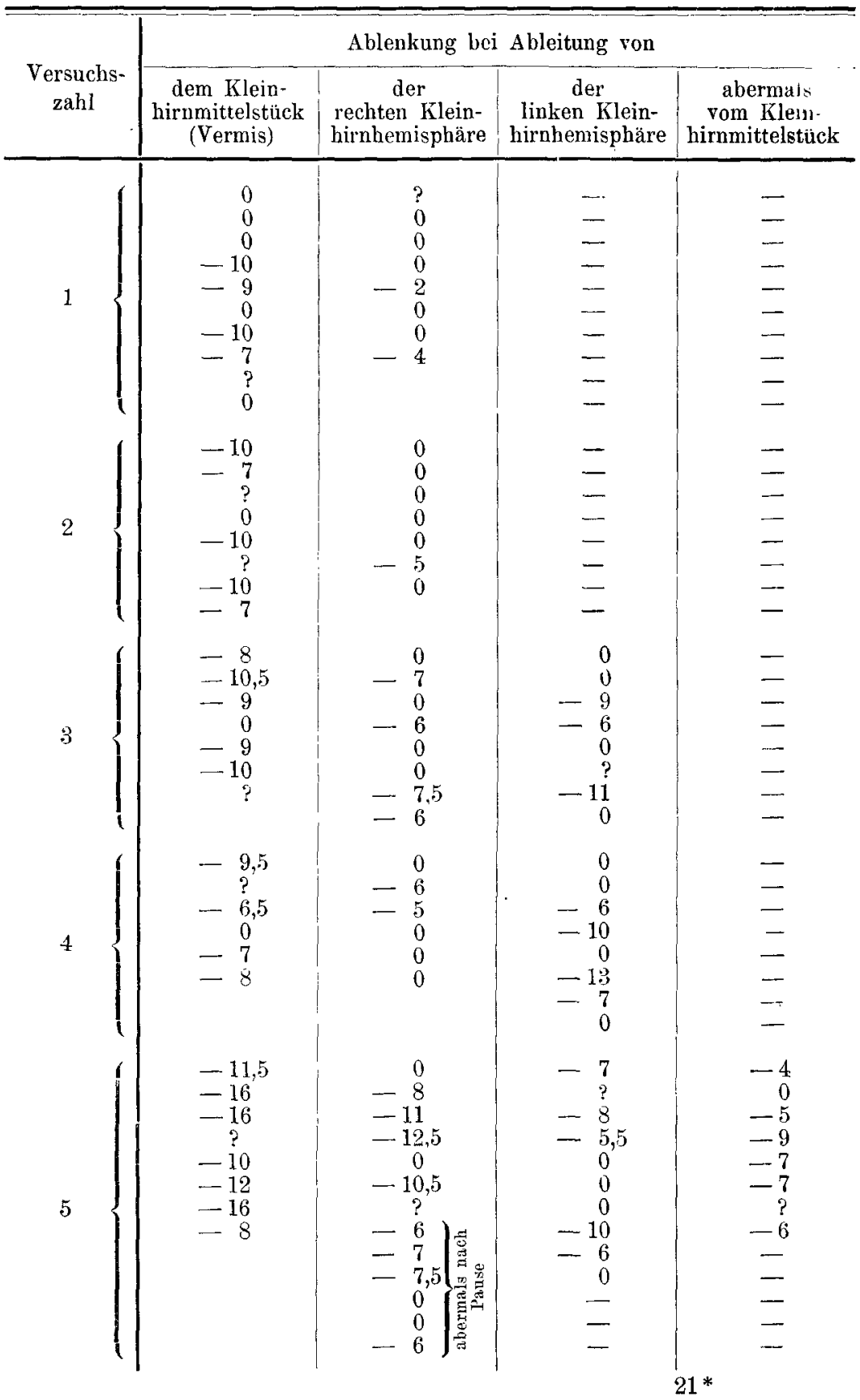


302 A. Beck und G. Bikeles: Versuche über die sensor. Funktion etc.

\begin{tabular}{|c|c|c|c|c|}
\hline \multirow[b]{2}{*}{$\begin{array}{l}\text { Versuchs- } \\
\text { zahl }\end{array}$} & \multicolumn{4}{|c|}{ Ablenkung bei Ableitung von } \\
\hline & $\begin{array}{l}\text { dem Klein- } \\
\text { hirnmittelstück } \\
\text { (Vermis) }\end{array}$ & $\begin{array}{l}\text { der } \\
\text { rechten Klein- } \\
\text { hirnhemisphäre }\end{array}$ & $\begin{array}{c}\text { der } \\
\text { linken Klein- } \\
\text { hirnhemisphäre }\end{array}$ & $\begin{array}{c}\text { abermals } \\
\text { vom Klein- } \\
\text { hirnmittelstück }\end{array}$ \\
\hline 6 & $\begin{array}{r}-6 \\
-10 \\
-\quad 7 \\
-13 \\
-7 \\
- \\
- \\
= \\
-\end{array}$ & $\begin{array}{r}0 \\
-\quad 6 \\
0 \\
? \\
0 \\
? \\
- \\
- \\
- \\
-\end{array}$ & $\begin{array}{r}0 \\
-\quad 2 \\
-\quad 8 \\
-8 \\
0 \\
-\quad 7 \\
-11 \\
0 \\
- \\
-\end{array}$ & $\begin{array}{r}0 \\
0 \\
-\quad 8 \\
-12 \\
-\quad 9 \\
-\quad 11 \\
-\quad 9 \\
-\quad 2 \\
-\quad 8 \\
-\quad 4\end{array}$ \\
\hline 7 & $\begin{array}{r}-8 \\
-\quad 8 \\
-\quad 5 \\
0 \\
-\quad 0 \\
-\quad 6 \\
-\quad 9 \\
-\quad 7\end{array}$ & $\begin{array}{r}0 \\
0 \\
? \\
0 \\
-\quad 5 \\
0 \\
-\quad 5 \\
0\end{array}$ & $\begin{array}{r}? \\
0 \\
-\quad 6 \\
0 \\
-\quad 5 \\
0 \\
? \\
0\end{array}$ & $\begin{array}{c}-7 \\
0 \\
-\quad 7 \\
-\quad 8 \\
-5,5 \\
- \\
-\end{array}$ \\
\hline 8 & $\begin{array}{c}-20 \\
-\quad 6 \\
-\quad 7,5 \\
-\quad 4 \\
-\quad 7 \\
0 \\
- \\
-\end{array}$ & $\begin{array}{r}9 \\
? \\
0 \\
0 \\
-\quad 4 \\
-\quad 9 \\
? ? \\
-11\end{array}$ & $\begin{array}{c}-5 \\
0 \\
-10,5 \\
? \\
-3 \\
0 \\
0 \\
- \\
-\end{array}$ & $\begin{array}{r}-\quad 4 \\
0 \\
-\quad 5 \\
-\quad 9 \\
-\quad 7 \\
-\quad 7 \\
-\quad 6\end{array}$ \\
\hline
\end{tabular}

als am Vermis posterior; in zwei Versuchen war jedenfalls ein ausgesprochener Unterschied in der Häufigkeit der Aktionsströme zu Gunsten des Mittelstückes zu konstatieren.

Dabei wurde in vier Versuchen nach stattgehabter Ableitung vom Lobulus paramedianus neuerdings vom Lobus medianus post. (Vermis post.), abgeleitet, und zwar mit durchgehends deutlich besserem Resultate als bei Ableitung vom Lobulus paramedianus. 